\title{
Hypoxia downregulates the expression of cell surface MICA without increasing soluble MICA in osteosarcoma cells in a HIF-1 $\alpha$-dependent manner
}

\author{
NAOKO YAMADA $^{1}$, KOJI YAMANEGI ${ }^{1}$, HIDEKI OHYAMA ${ }^{1}$, MASAKI HATA $^{1}$, KEIJI NAKASHO ${ }^{1}$, \\ HIROYUKI FUTANI $^{2}$, HARUKI OKAMURA ${ }^{3}$ and NOBUYUKI TERADA ${ }^{1}$ \\ Departments of ${ }^{1}$ Pathology, ${ }^{2}$ Orthopedic Surgery and ${ }^{3}$ Tumor Immunology and Cell Therapy, \\ Hyogo College of Medicine, Nishinomiya, Hyogo 663-8501, Japan
}

Received June 8, 2012; Accepted August 9, 2012

DOI: $10.3892 /$ ijo.2012.1630

\begin{abstract}
Tumor cells express NKG2D ligands on their cell surface, which are the ligands of the activating receptor, NKG2D, that is expressed on the surface of NK cells. The binding of NK cells to tumor cells through the interaction of NKG2D and its ligands induces the cytolysis of the tumor cells. In the present study, we investigated the effects of hypoxia on the expression of NKG2D ligands on the surface of human osteosarcoma cells using three cell lines. To produce hypoxic and normoxic conditions, the osteosarcoma cell lines were cultured under 1 and $20 \% \mathrm{O}_{2}$ conditions, respectively. The osteosarcoma cells expressed NKG2D ligands such as MHC class I-related chain molecules A and B (MICA and MICB) and the UL16-binding proteins 1, 2 and 3 (ULBP 1, 2 and 3). MICA was the most frequently expressed NKG2D ligand in the osteosarcoma cells. Hypoxia decreased the expression of cell surface MICA only without increasing the secretion of soluble MICA, which is produced by proteolytic cleavage of cell surface MICA. Hypoxia consistently decreased the susceptibility of the osteosarcoma cells to the cytotoxicity of the NK cells. Hypoxia induced the expression of hypoxia-inducible factor- $1 \alpha$ (HIF-1 $\alpha)$, and knockdown of the expression of HIF-1 $\alpha$ using small interfering RNA increased the expression of cell surface MICA and concomitantly increased the level of soluble MICA. Hypoxia decreased the production of nitric oxide (NO) metabolites (nitrite and nitrate), thus, indicating a decreasing effect on NO production. However, a NO donor, NOC18, decreased the expression of cell surface MICA without any apparent
\end{abstract}

Correspondence to: Dr Naoko Yamada, Department of Pathology, Hyogo College of Medicine, 1-1 Mukogawa-cho, Nishinomiya, Hyogo 663-8501, Japan

E-mail: ynaoko@hyo-med.ac.jp

Key words: hypoxia, MHC class I-related chain molecule, hypoxiainducing factor- $1 \alpha$, nitric oxide, soluble MHC class I-related chain molecule A, osteosarcoma effects on the expression of HIF-1 $\alpha$ under both hypoxic and normoxic conditions. The present results indicate that hypoxia downregulates the expression of cell surface MICA without increasing the level of soluble MICA in a HIF-1 $\alpha$-dependent manner and suggest that the effects of hypoxia are not linked to the hypoxia-induced reduction of NO production.

\section{Introduction}

Hypoxia is a common condition found within a variety of solid tumors. Adaptive responses of tumor cells to hypoxia develop the malignant phenotypes of the tumor cells, which promotes angiogenesis, invasion, metastasis and resistance to chemotherapy and radiotherapy (1-3). Hypoxia-inducing factor-1 (HIF-1) plays a pivotal role in the adaptive responses of tumor cells to hypoxia. HIF-1, which is a heterodimer with an oxygensensitive HIF-1 $\alpha$ subunit and a constitutively expressed HIF-1 $\beta$ subunit, binds the hypoxia-responsive element and activates the transcription of target genes (4-6). Under normoxia, HIF-1 $\alpha$ is rapidly degraded by the ubiquitin-proteasome pathway; however, under hypoxia, HIF-1 $\alpha$ is stabilized and accumulates in cells $(5,7)$.

Tumor cells express NKG2D ligands on their cell surface, which are ligands of an activating receptor, NKG2D, that is expressed on the cell surface of cytotoxic immune cells, such as NK, $\gamma \delta^{+} \mathrm{T}$ and $\mathrm{CD} 8^{+} \alpha \beta^{+} \mathrm{T}$ cells $(8,9)$. In humans, there are two families of NKG2D ligands, the MHC class I-related chain molecules A and B (MICA and MICB) and the UL16-binding proteins (ULBP) (8). The binding of an NKG2D receptor to its ligand activates $\mathrm{NK}$ and $\gamma \delta^{+} \mathrm{T}$ cells and co-stimulates tumor-antigen-specific $\mathrm{CD}^{+} \alpha \beta^{+} \mathrm{T}$ cells $(8,9)$. Therefore, the NKG2D ligands on the surface of tumor cells are important for the cytotoxicity of immune cells. On the other hand, tumor cells produce soluble forms of NKG2D ligands by proteolytic cleavage of their extracellular domains $(8,10-13)$. Soluble forms of NKG2D ligands interfere with the binding of NKG2D ligands on the surface of tumor cells to NKG2D receptors on the surface of cytotoxic immune cells, and the binding of soluble NKG2D ligands to NKG2D receptors downregulates the NKG2D receptors on the surface of cytotoxic immune cells (8,13-16). Therefore, a decrease in the expression of NKG2D ligands on 
the surface of tumor cells and an increase in the secretion of soluble NKG2D ligands attenuate the susceptibility of tumor cells to cytotoxic immune cells.

Regarding the effects of hypoxia on immune surveillance, there are many studies on the hypoxia-induced inhibition of cytotoxic activity of immune cells (17); however, only a few studies have addressed the effects of hypoxia on immune escape by tumor cells $(18,19)$. In this study, we examined the effects of hypoxia on the expression of the cell surface NKG2D ligands of osteosarcoma cells, the secretion of soluble NKG2D ligands and the role of HIF-1 $\alpha$ in the hypoxia-induced effects. Several studies have previously shown such hypoxia-induced effects to be produced by the hypoxia-induced inhibition of the nitric oxide (NO) signaling pathway (18-22) and that NO modulates hypoxia-induced cellular events $(23,24)$. Therefore, we also examined the role of $\mathrm{NO}$ in hypoxia-induced effects.

\section{Materials and methods}

Cell culture. U2-OS human osteosarcoma cells and NK-92 human natural killer cells were purchased from the American Type Culture Collection (Rockville, MD, USA). HOS and SaOS-2 human osteosarcoma cells were purchased from the Riken BRC Cell Bank (Tsukuba, Ibaragi, Japan). All osteosarcoma cells were maintained in Dulbecco's modified Eagle's medium (DMEM) (Invitrogen, Carlsbad, CA, USA) containing $10 \%$ fetal bovine serum (FBS) (PAA, Pasching, Oberosterreich, Austria). Human NK cells were cultured in Minimum Essential Medium Alpha Medium (Invitrogen) containing $12.5 \%$ horse serum (MP Biomedicals, Solon, OH, USA), $12.5 \%$ FBS, $0.2 \mathrm{mM}$ inositol (Sigma, St. Louis, MO, USA), $0.1 \mathrm{mM} 2$-mercaptoethanol (Wako, Osaka, Japan), $0.02 \mathrm{mM}$ folic acid (Sigma) and $100 \mathrm{U} / \mathrm{ml}$ recombinant human IL-2 (Peprotech, Rocky Hill, NJ, USA). For the cell cultures under $20 \% \mathrm{O}_{2}$ conditions, the cells were cultured in a humidified atmosphere of $5 \% \mathrm{CO}_{2}$ in air at $37^{\circ} \mathrm{C}$. For the cell cultures under $1 \% \mathrm{O}_{2}$ conditions, the cells were cultured in a humidified atmosphere of $1 \% \mathrm{O}_{2}$ and $5 \% \mathrm{CO}_{2}$ in $\mathrm{N}_{2}$ at $37^{\circ} \mathrm{C}$ using a $\mathrm{CO}_{2}$ Multi GAS incubator (Astec, Fukuoka, Japan).

Growth of the osteosarcoma cells. Cells seeded at $1 \times 10^{3}$ cells per well of 96-well tissue culture plates were cultured in $100 \mu 1$ of DMEM containing 10\% FBS under either 20 or $1 \% \mathrm{O}_{2}$ conditions for $72 \mathrm{~h}$. The number of viable cells in each well was estimated using a Cell counting kit-8 (Dojindo, Kumamoto, Japan).

Flow cytometry. The cells were harvested using a brief treatment with $0.25 \%$ trypsin and $0.1 \mathrm{mM}$ EDTA in phosphate-buffered saline (PBS), pH 7.4, resuspended in an ice-cold FACS buffer (PBS containing 2\% FBS) and incubated with either phycoerythrin (PE)-labeled mouse anti-human MICA $\mathrm{mAb}$ (monoclonal IgG antibody) (100-fold dilution), mouse anti-human MICB mAb (100-fold dilution), mouse anti-human ULBP1 mAb (100-fold dilution), mouse anti-human ULBP2 $\mathrm{mAb}$ (100-fold dilution) or mouse anti-human ULBP3 mAb (100-fold dilution) on ice for $30 \mathrm{~min}$. As a control, cells were incubated with mouse IgG. When incubated with an unlabelled $\mathrm{mAb}$, the cells were washed with FACS buffer and further incubated with the PE-labeled goat anti-mouse IgG (200-fold dilution) on ice for $30 \mathrm{~min}$. The cells were then washed with FACS buffer and analyzed using a FACSCalibur flow cytometer (Becton Dickinson, Mountain View, CA, USA) and the percentage of positive cells was determined. Cells $(10,000)$ were used for each flow cytometric analysis. All antibodies and mouse IgG used for flow cytometry were purchased from R\&D Systems (Minneapolis, MN, USA).

Western blot analysis. The cultured cells were homogenized with lysis buffer containing $50 \mathrm{mM}$ Tris-HCl, $\mathrm{pH} 7.5,150 \mathrm{mM}$ $\mathrm{NaCl}, 0.1 \%$ SDS, $1 \%$ Triton X-100, $1 \%$ sodium deoxycholate and a cocktail of protease inhibitors (Complete) (Roche, Penzberg, Bavaria, Germany) using a sonicator (Sonics \& Materials, Newtown, CT, USA). An aliquot of the cell homogenate containing $25 \mu \mathrm{g}$ of proteins was boiled in a SDS sample buffer (New England BioLabs, Ipswich, MA, USA) and subjected to electrophoresis in a denaturing 5-10\% SDS-polyacrylamide gradient gel (Atto, Tokyo, Japan). The separated proteins were transferred onto a polyvinylidene difluoride membrane (Fine Trap) (Nihon Eido, Tokyo, Japan). The membranes were blocked with 5\% non-fat dry milk in PBS containing $0.1 \%$ Tween-20 and incubated with primary mouse anti-human HIF-1 $\alpha$ mAb (2,000-fold dilution) (BD Biosciences, Franklin Lakes, NJ, USA), rabbit anti-human MICA pAb (polyclonal antibody) (2,000-fold dilution) (GeneTex, Irvine, CA, USA) and rabbit anti-GAPDH pAb (4,000-fold dilution) (GeneTex) or rabbit anti-human $\beta$-actin pAb (4,000-fold dilution) (Thermo Fisher Scientific, Fremont, CA, USA) at room temperature for $1 \mathrm{~h}$. Proteins bound to the primary antibodies were detected using a horseradish peroxidase (HRP)-conjugated goat antirabbit IgG or HRP-conjugated goat anti-mouse IgG (Santa Cruz Biotechnology, Santa Cruz, CA, USA) and ECL Western Blotting Detection reagents (GE Healthcare, Little Chalfont, UK).

Quantitative real-time reverse transcription-polymerase chain reaction (real-time RT-PCR). Total-RNA was extracted from the cells using a TRIzol reagent (Invitrogen) and DNase (1 U/pl) (Wako). The cDNAs were synthesized and amplified using an RNA-direct ${ }^{\mathrm{TM}}$ SYBR-Green Real-time PCR Master mix (Toyobo, Osaka, Japan) and the following specific primers: sense, 5'-AGATTTTGGCAGCAACGACA-3' (1637-1656) and antisense, 5'-GCGGTGGGTAATGGAGACAT-3' (1752-1771) for the HIF-1 $\alpha$ cDNA, sense, 5'-ACTGCTTGAGCCGCTG AGA-3' (2-20) and antisense, 5'-GAGGTGCAAAAGGGAA GATGC-3' (74-94) for MICA cDNA, sense, 5'-GGGGCGC AGGTGACTAAAT-3' (33-51) and antisense, 5'-CCTACGTCG CCACCTTCTCA-3' (93-112) for MICB cDNA, and sense, 5'-CGTGGCTAAACAGGTACTGCTG-3' (88-109) and antisense, 5'-GGAGGTTTGCCAGGTA-3' (177-197) for ribosomal protein L13a (RPL13a) cDNA. The real-time PCR was performed under the PCR conditions: 45 cycles at $95^{\circ} \mathrm{C}$ for $15 \mathrm{sec}$, at $62^{\circ} \mathrm{C}$ for $15 \mathrm{sec}$ and at $74^{\circ} \mathrm{C}$ for $35 \mathrm{sec}$ using an ABI PRIAM 7900HT (Applied Biosystems, Foster, CA, USA). The amount of mRNA in each gene was corrected by the amount of mRNA of RPL13a in a corresponding sample.

Cytotoxicity assay. Osteosarcoma cells (T, target cells; $2 \times 10^{4}$ cells) suspended in $100 \mu \mathrm{l}$ of DMEM containing $10 \%$ FBS were mixed with NK cells (E, effector cells) suspended in $100 \mu 1$ of DMEM containing 10\% FBS at various T:E ratios (1/10-1/2.5) 
and placed into wells of 96 -well plates. The cells were incubated at $37^{\circ} \mathrm{C}$ for $4 \mathrm{~h}$ in a humidified atmosphere of $5 \% \mathrm{CO}_{2}$ in air. The cytotoxicity assay was performed using a Cytotoxicity Detection kitplus (Roche).

Assay of soluble MICA and MICB. The levels of soluble MICA and MICB in culture medium were determined using DuoSet ELISA Development kits for MICA and MICB (R\&D Systems).

Assay of nitrite and nitrate. Osteosarcoma cells seeded at $5 \times 10^{5}$ cells per well in 6-well tissue culture plates were cultured in $5 \mathrm{ml}$ of medium for $24 \mathrm{~h}$. Then, the culture medium was changed to a medium with or without $100 \mu \mathrm{M}$ NOC18 [1-Hydroxy-2oxo-3,3-bis(2-aminoethyl)-1-triazene] (Dojindo) and cultured for $24 \mathrm{~h}$ under either 20 or $1 \% \mathrm{O}_{2}$ conditions. After the culture was completed, the medium was collected and the amount of nitrite and nitrate in the culture medium was measured using a $\mathrm{NO}_{2} / \mathrm{NO}_{3}$ Assay kit CII (Dojindo).

RNA interference. Small interfering RNA (siRNA) for human HIF-1 $\alpha$ [siHIF-1 (siRNA ID No. 6539 ) or siHIF-2 (siRNA ID No. 6541)] and control siRNA (Catalog No. 4390843) were purchased from Invitrogen. The transfection of siRNA into the osteosarcoma cells was performed using a Lipofectamine RNAiMAX (Invitrogen) according to the manufacturer's instructions. Briefly, HIF-1 $\alpha$ siRNA or control siRNA and a Lipofectamine RNAiMAX were mixed in $100 \mu$ l of Opti-MEM medium (Invitrogen) in wells of 24-well tissue culture plates. Thereafter, $2 \times 10^{4}$ osteosarcoma cells suspended in $500 \mu \mathrm{l}$ of Opti-MEM medium were added into each well, which resulted in a final siRNA concentration of $10 \mathrm{nM}$, and the cells were cultured for $24 \mathrm{~h}$. Then, the medium was changed to $1 \mathrm{ml}$ of DMEM containing 10\% FBS and the cells were cultured for another $24 \mathrm{~h}$ under either 20 or $1 \% \mathrm{O}_{2}$ conditions. After the culture was completed, the medium and the cells were collected for assay of soluble MICA and soluble MICB in the medium and analyses of the amounts of HIF- $1 \alpha$ mRNA, HIF-1 $\alpha$ protein and MICA.

Statistical analysis. The data of two groups were analyzed using Student's t-test, and the data of three or more groups were analyzed using the Bonferroni multiple comparison test. A P-value of $<0.05$ was considered to be significant.

\section{Results}

Effects of hypoxia on the growth of osteosarcoma cell lines. HOS, U2-OS and SaOS-2 cells were cultured in normoxic $\left(20 \% \mathrm{CO}_{2}\right)$ and hypoxic $\left(1 \% \mathrm{O}_{2}\right)$ conditions for $72 \mathrm{~h}$. When the average viable cell number in the normoxic conditions was expressed as 1 , the viable cell numbers (means $\pm \mathrm{SE}, \mathrm{n}=12$ ) in the hypoxic conditions were $1.02 \pm 0.06$ for HOS cells, $0.99 \pm 0.05$ for U2-OS cells and $0.98 \pm 0.04$ for SaOS-2 cells, respectively, and hypoxia did not affect the growth of the osteosarcoma cells for at least $72 \mathrm{~h}$.

Effects of hypoxia on cell surface NKG2D ligands and their soluble forms. Osteosarcoma cells were cultured for $72 \mathrm{~h}$ in normoxic and hypoxic conditions, and the expression of cell surface NKG2D ligands and the amounts of their soluble forms in the medium were examined. As shown in Fig. 1, three osteosarcoma cell lines expressed cell surface MICA and ULBP2 in high percentages, whereas cell surface MICB, ULBP1 and ULBP3 were expressed in very low percentages. Hypoxia decreased the expression of cell surface MICA significantly; however, the expression of ULBP2 did not decrease. Hypoxia increased the expression of cell surface MICB and ULBP1 in U2-OS cells a little; however, it did not affect the expression of cell surface MICB or ULBP1 in HOS and SaOS-2 cells. Hypoxia did not affect the expression of ULBP3 in any of the three osteosarcoma cell lines.

The amount of soluble MICA detected in the medium was much lower than that of soluble MICB. Hypoxia did not affect the amounts of either soluble MICA or soluble MICB in the medium (Fig. 2).

Effects of hypoxia on the susceptibility of osteosarcoma cell lines to NK cells. Since hypoxia decreased the expression of cell surface MICA in the osteosarcoma cells, the effect of this decrease on the susceptibility of osteosarcoma cells to the cytotoxicity of NK cells was examined (Fig. 3). Culture of three osteosarcoma cell lines for $72 \mathrm{~h}$ under hypoxic conditions decreased the susceptibility of all three osteosarcoma cell lines to NK cells.

Role of hypoxia-induced expression of HIF-1 $\alpha$. It has been reported that hypoxia induces the accumulation of HIF- $1 \alpha$ proteins and that hypoxia-induced cellular events are mediated by HIF-1 $\alpha(4,5)$. Therefore, the role of HIF-1 $\alpha$ in the hypoxiainduced decrease in the expression of cell surface MICA was investigated.

Hypoxia for $24 \mathrm{~h}$ increased the amount of HIF- $1 \alpha$ and decreased the amount of MICA in all three osteosarcoma cell lines (Fig. 4A). However, hypoxia did not increase the amount of mRNA of both HIF-1 $\alpha$ and MICA (Fig. 4B).

To determine whether the hypoxia-induced decrease in the amount of MICA is mediated by HIF- $1 \alpha$, we knocked down HIF-1 $\alpha$ mRNA using siRNA (siHIF-1 or siHIF-2). The HIF-1 $\alpha$ siRNA transfection decreased the HIF-1 $\alpha$ mRNA expression (Fig. 5) and increased the MICA mRNA expression in the three osteosarcoma cell lines cultured under both normoxic and hypoxic conditions for $24 \mathrm{~h}$ (Fig. 5). A western blot analysis showed that the HIF-1 $\alpha$ siRNA transfection decreased the amount of HIF- $1 \alpha$ proteins and increased the amount of MICA proteins in osteosarcoma cells cultured under both normoxic and hypoxic conditions (Fig. 6A). A flow cytometric analysis showed that the increase in the amount of MICA proteins in the osteosarcoma cell lines reflected the increase in cell surface MICA (Fig. 6B). The HIF-1 $\alpha$ siRNA transfection increased the amount of soluble MICA in the medium of all three osteosarcoma cell lines cultured under hypoxic conditions and in the medium of U2-OS cells cultured under normoxic conditions, while the amount of soluble MICA in the medium of HOS and SaOS-2 cells cultured under normoxic conditions was undetectable (Fig. 6C).

Participation of $N O$ in the hypoxia-induced decrease in the MICA expression. The participation of NO in the hypoxiainduced decrease in the MICA expression was examined. First, 
A
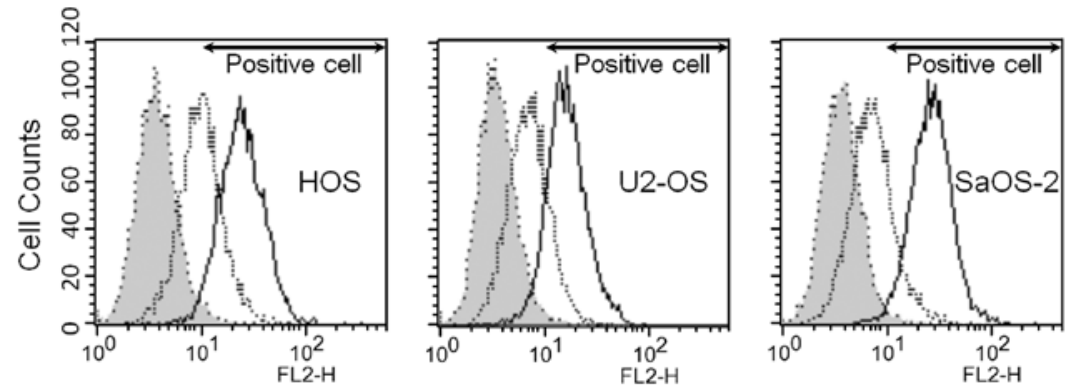

$\mathrm{B}$
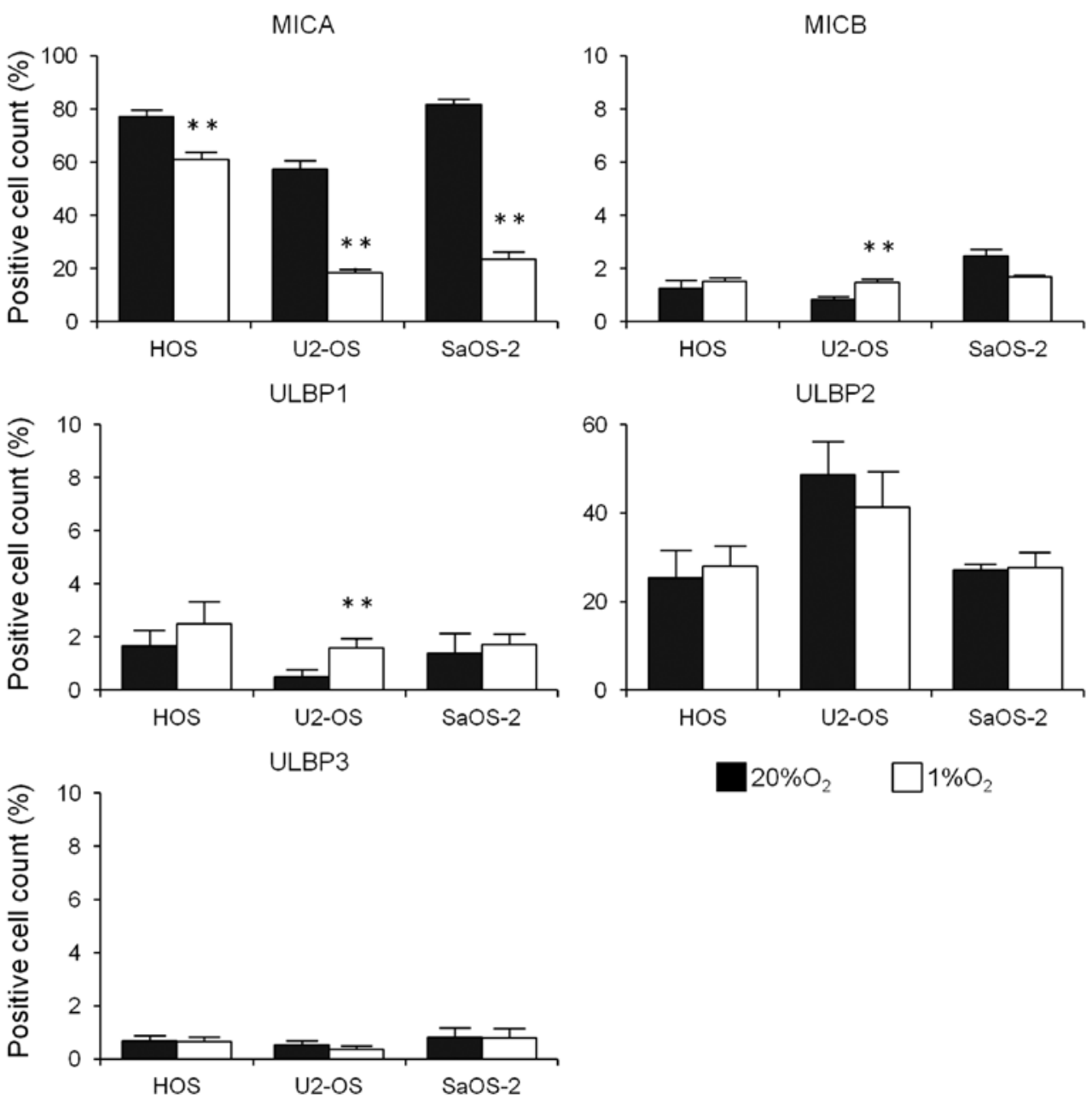

Figure 1. Effects of hypoxia on the expression of cell surface NKG2D ligands. Three osteosarcoma cell lines $\left(2 \times 10^{5} \mathrm{cells} / 10 \mathrm{ml}\right.$ medium/10-cm dish) were cultured for $72 \mathrm{~h}$ under either normoxic or hypoxic conditions. (A) Representative flow cytometric profiles of cell surface MICA. The shadow profile indicates a control profile of cells incubated with mouse IgG. The dashed and solid lines indicate the profiles of cells cultured under hypoxic and normoxic conditions, respectively. Positive cells are indicated by the horizontal bar. (B) Percentages of cells expressing MICA, MICB and ULBP 1,2 and 3. Each bar shows the mean \pm SE of five or six dishes. ${ }^{* *} \mathrm{P}<0.01$, a significant difference from cells cultured under normoxic conditions.

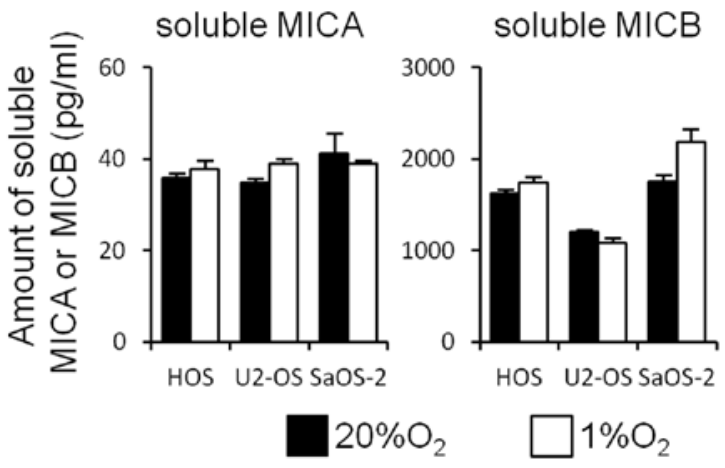

osteosarcoma cells were cultured for $24 \mathrm{~h}$ under normoxic and hypoxic conditions and the amount of $\mathrm{NO}$ metabolites $\left(\mathrm{NO}_{2}\right.$ and $\mathrm{NO}_{3}$ ) in the medium was assayed. Hypoxia decreased the amount of NO metabolites in the medium (Fig. 7A). Next, the

Figure 2. Effects of hypoxia on the secretion of soluble MICA or MICB. Three osteosarcoma cell lines ( $2 \times 10^{5} \mathrm{cells} / 8 \mathrm{ml}$ medium $/ 10$-cm dish) were cultured for $72 \mathrm{~h}$ under either normoxic or hypoxic conditions and the amounts of soluble MICA and MICB in the medium were assayed. Each bar shows the mean $\pm \mathrm{SE}$ of five or six dishes. 

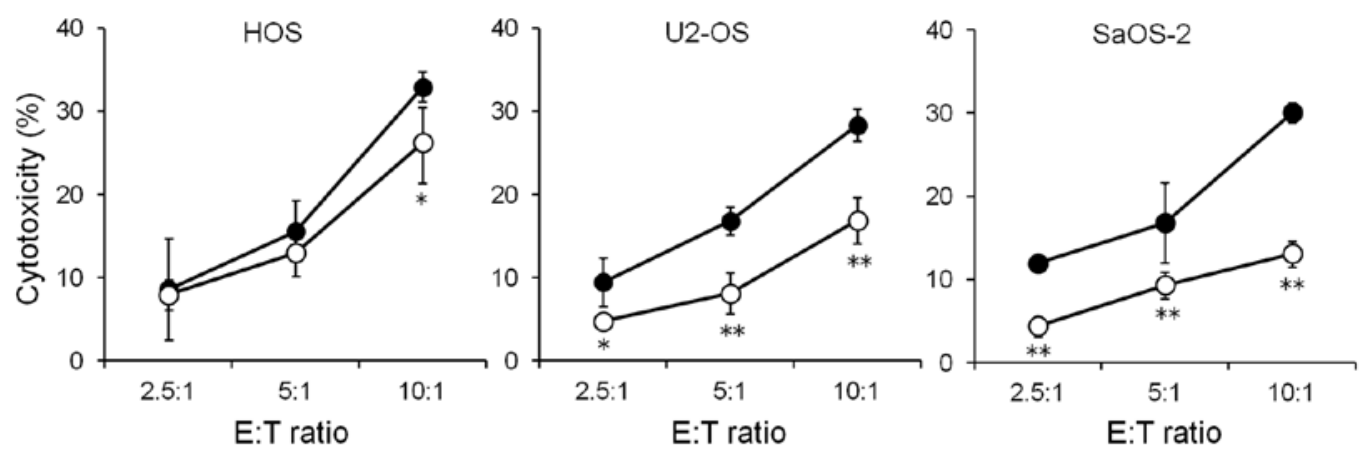

$20 \% \mathrm{O}_{2} \quad \mathrm{O}_{1} \% \mathrm{O}_{2}$

Figure 3. Effects of hypoxia on the susceptibility of osteosarcoma cells to NK cell-mediated cytotoxicity. Three osteosarcoma cell lines were cultured for $72 \mathrm{~h}$ under either normoxic or hypoxic conditions and the cells ( $\mathrm{T}$, target cells) were incubated with NK-92 cells (E, effector cells) at various E:T ratios. Each point represents a mean \pm SE of six wells. ${ }^{* *} \mathrm{P}<0.01$ and ${ }^{*} \mathrm{P}<0.05$, a significant difference from the values of cells cultured under normoxic conditions.

A

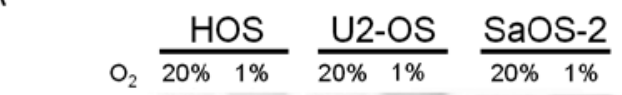

HIF-1a

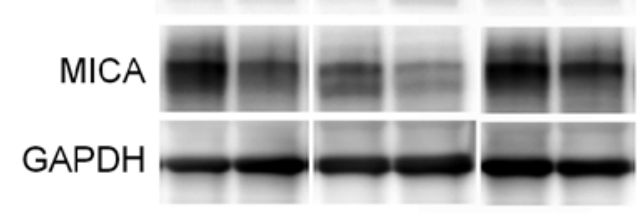

B
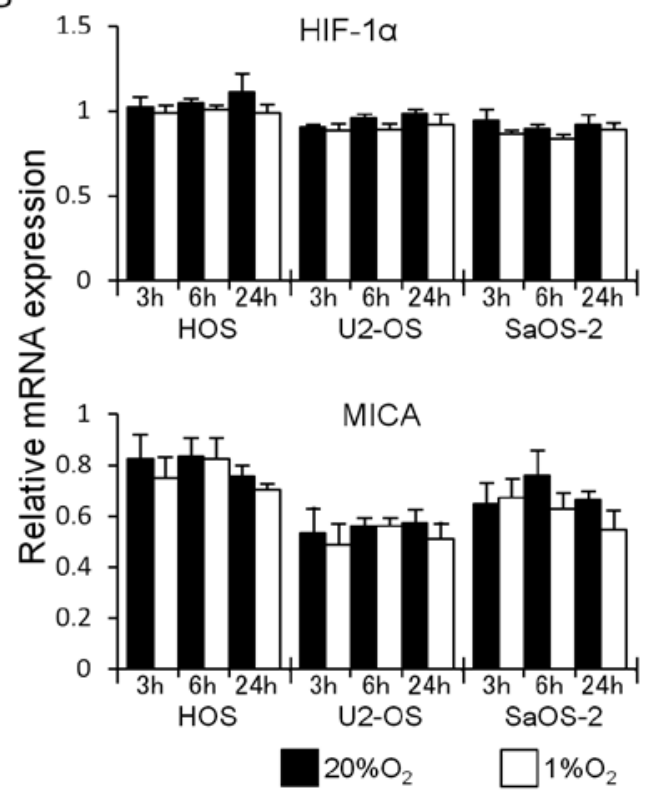

Figure 4. Effects of hypoxia on the expression of HIF-1 $\alpha$ and MICA and their mRNAs. Osteosarcoma cells were cultured for $24 \mathrm{~h}$ under either normoxic or hypoxic condition. (A) A western blot analysis of the expression of HIF-1 $\alpha$ and MICA after culture for $24 \mathrm{~h}$. (B) The real-time PCR analysis of HIF-1 $\alpha$ and MICA mRNAs. Each bar represents the mean \pm SE of four wells. The average expression level of the cells before the culture is expressed as 1 .

effects of the NO donor (NOC18) on the amounts of HIF-1 $\alpha$ and MICA proteins in the osteosarcoma cell lines were exam-

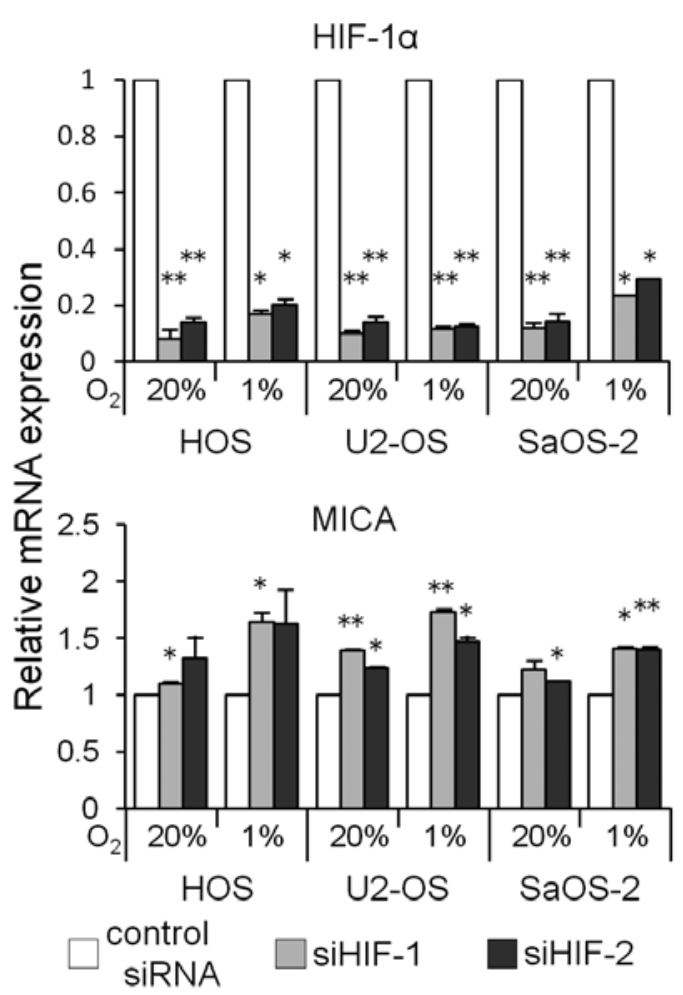

Figure 5. Effects of transfection of siRNA for HIF-1 $\alpha$ into osteosarcoma cells on the expression of HIF-1 $\alpha$ and MICA mRNAs. Osteosarcoma cells were transfected with either siRNA for HIF-1 $\alpha$ (siHIF-1 or siHIF-2) or control siRNA. Then, these cells $\left(2 \times 10^{4}\right.$ cells in $1 \mathrm{ml}$ medium/well) were cultured for $24 \mathrm{~h}$ under either normoxic or hypoxic conditions. Thereafter, the amounts of HIF-1 $\alpha$ and MICA mRNAs were estimated using real-time PCR. Each bar represents the mean $\pm \mathrm{SE}$ of four experiments. The average expression level for cells transfected with control siRNA in each experiment is expressed as 1. ${ }^{* *} \mathrm{P}<0.01$ and ${ }^{*} \mathrm{P}<0.05$, a significant difference from the values of cells transfected with the control siRNA.

ined. The NO donor increased the amount of $\mathrm{NO}$ metabolites markedly (Fig. 7A). The NO donor did not affect the amount of HIF-1 $\alpha$ proteins under either normoxic or hypoxic conditions; however, the amount of MICA under both normoxic and hypoxic conditions was observed to decrease. 
A
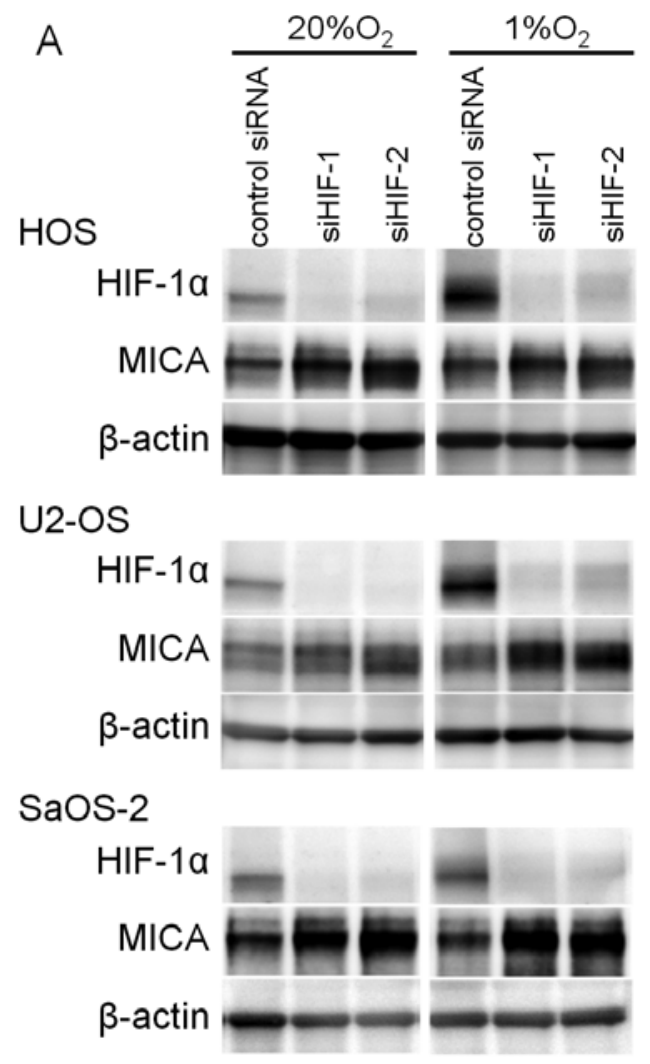
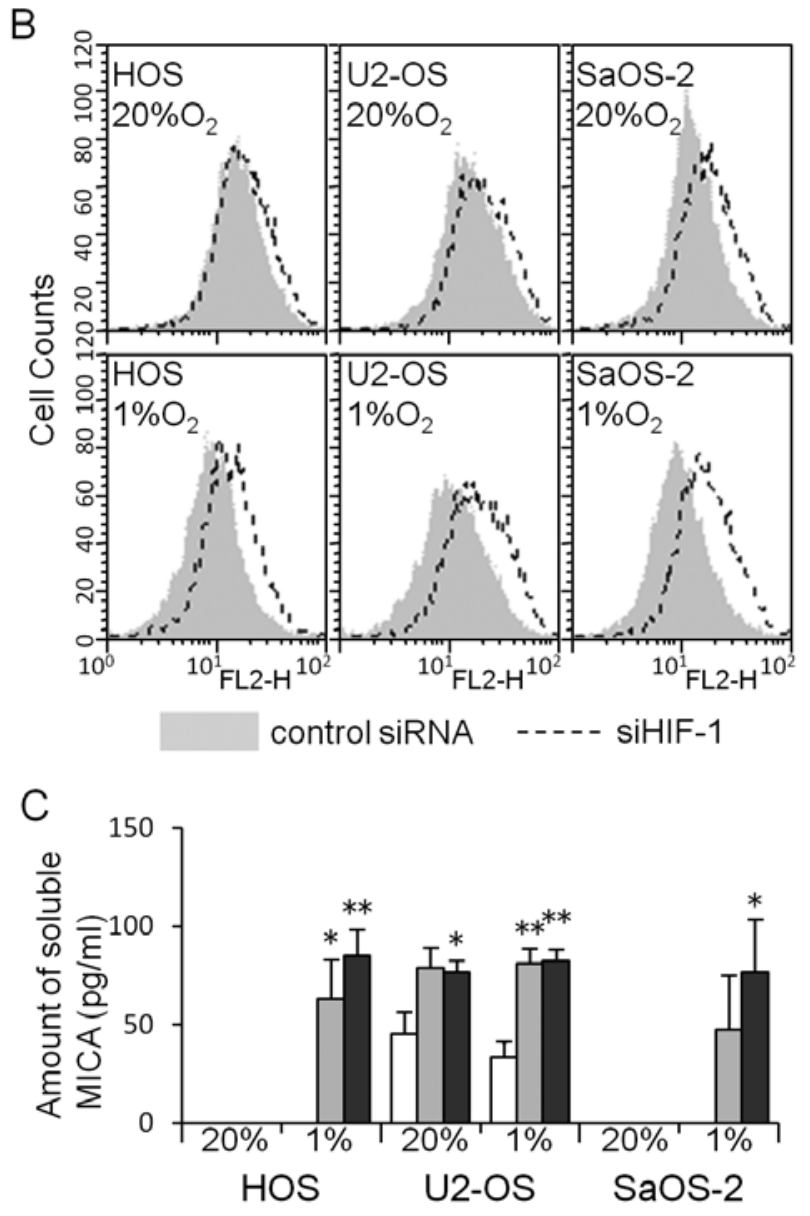

Figure 6. Effects of transfection of siRNA for HIF-1 $\alpha$ into osteosarcoma cells on the expression of HIF-1 $\alpha$ and MICA and the secretion of soluble MICA. Osteosarcoma cells were transfected with either siRNA for HIF-1 $\alpha$ (siHIF-1 or siHIF-2) or control siRNA. Then, these cells ( $2 \times 10^{4}$ cells/1 ml medium/well) were cultured for $24 \mathrm{~h}$ under either normoxic or hypoxic conditions. The expression of HIF-1 $\alpha$ and MICA was examined and the amount of soluble MICA in the medium was assayed. (A) The western blot analysis. (B) The flow cytometric analysis of the expression of MICA. The representative profiles are presented. The shadow profile indicates cells transfected with control siRNA and the dashed line indicates a profile of cells transfected with siHIF-1. (C) The amount of soluble MICA in the medium. Each bar represents the mean \pm SE of four wells. ${ }^{* *} \mathrm{P}<0.01$ and ${ }^{*} \mathrm{P}<0.05$, a significant difference from the value of cells transfected with control siRNA.

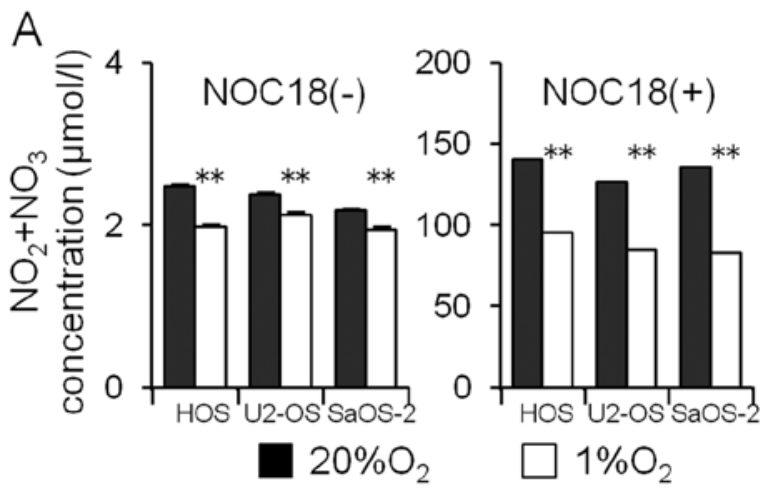

Figure 7. Role of nitric oxide (NO) on the expression of HIF-1 $\alpha$ and MICA. Osteosarcoma cell lines $\left(5 \times 10^{5}\right.$ cells in $5 \mathrm{ml}$ medium/well) were cultured for $24 \mathrm{~h}$ under either normoxic or hypoxic conditions in the presence or absence of $\mathrm{NOC} 18(100 \mu \mathrm{M})$. At the end of the culture, the concentration of nitrite $\left(\mathrm{NO}_{2}\right)$ plus nitrate $\left(\mathrm{NO}_{3}\right)$ in the medium was measured and the expression of HIF-1 $\alpha$ and MICA in osteosarcoma cells was examined using a western blot analysis. (A) The concentration of $\mathrm{NO}_{2}+\mathrm{NO}_{3}$ in the medium. Each bar represents the mean \pm SE of 4 dishes. ${ }^{* *} \mathrm{P}<0.01$, a significant difference from the value of cells cultured in $20 \% \mathrm{O}_{2}$ conditions. (B) A western blot analysis of the expression of HIF-1 $\alpha$ and MICA.
B

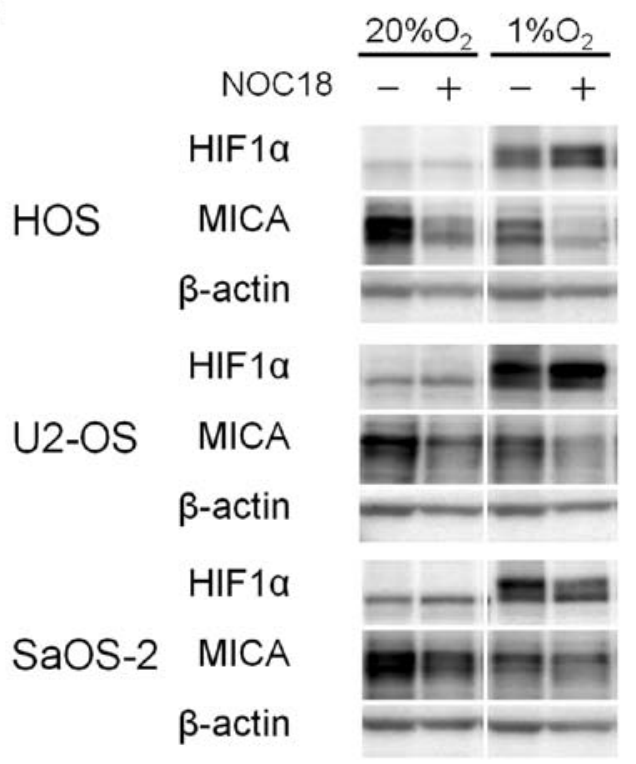




\section{Discussion}

Hypoxia downregulated the expression of MICA on the surface of osteosarcoma cells. Osteosarcoma cells secreted a relatively small amount of soluble MICA, compared to that of soluble $\mathrm{MICB}$, in the medium, and hypoxia did not increase the secretion of soluble MICA. Siemens et al (18) and Barsoum et al (19) have shown that hypoxia decreases the expression of MICA on the surface of prostatic and breast cancer cells and ascribes this decrease to the proteolytic cleavage of cell surface MICA by ADAM10 (a disintegrin and metalloproteinase 10). The tumor cells used for their experiments secreted a much larger amount of soluble MICA than osteosarcoma cells. Furthermore, hypoxia for $24 \mathrm{~h}$ did not affect the expression of ADAM10 mRNA in osteosarcoma cells (data not shown). Therefore, the difference between their results and ours may be due to the use of different tumor cells.

Hypoxia did not decrease the expression of cell surface NKG2D ligands, except for that of cell surface MICA. Since hypoxia did not affect the expression of MICA mRNA in spite of a decrease in cell surface MICA, the decrease in the expression of cell surface MICA may be ascribed to an attenuated translation of MICA mRNA or a rapid degradation of MICA in osteosarcoma cells under hypoxia. On the other hand, hypoxia showed only slight effects on the expression of cell surface MICB or the secretion of soluble MICB. Hypoxia for 3, 6 and $24 \mathrm{~h}$ did not affect the expression of MICB mRNA (data not shown). The mechanisms underlying the fact that hypoxia exerts different effects on cell surface MICA and other NKG2D ligands must be further investigated.

Hypoxia increased the expression of HIF-1 $\alpha$ without increasing the amount of HIF- $1 \alpha$ mRNA, as a result that is in agreement with those of previous reports $(5,7)$. Furthermore, the knockdown of HIF-1 $\alpha$ mRNA increased the expression of MICA mRNA and cell surface MICA and concomitantly increased the secretion of soluble MICA. These results show that the effects of hypoxia on the expression of cell surface MICA are mediated by HIF-1 $\alpha$. It has been reported that HIF-1 $\alpha$ upregulates the expression of MICA in human renal proximal tubular epithelial cells and cardiomyocytes $(25,26)$. The effects of HIF-1 $\alpha$ on the expression of cell surface MICA may be different between tumor cells and normal cells.

Several studies have shown that hypoxia-induced inhibition of NO production is linked to hypoxia-induced tumor invasion, metastasis, resistance to chemotherapy and shedding of cell surface MICA (18-22). In this study, hypoxia also induced the inhibition of NO production in osteosarcoma cells. However, under hypoxia the NO donor NOC18 decreased the expression of MICA in osteosarcoma cells. These results indicate that hypoxia-induced inhibition of NO production is not linked to the hypoxia-induced decrease in the expression of MICA in osteosarcoma cells.

Under both hypoxia and normoxia, NOC18 showed only slight effects on the expression of HIF-1 $\alpha$; however, NOC18 decreased the expression of MICA in osteosarcoma cells. Therefore, it is likely that NO modulates the hypoxia-induced $\mathrm{HIF}-1 \alpha$-dependent decrease in the expression of MICA in osteosarcoma cells.

The present and previous studies $(18,19)$ show that hypoxia decreases the susceptibility of tumor cells by reducing the expression of cell surface MICA, although the mechanisms of the reduction in the expression of cell surface MICA differ. Hypoxia has been reported to attenuate the antitumor activity of cytotoxic immune cells (17). Therefore, a hypoxic microenvironment seems to be more conducive to allowing tumor cells to escape from immune surveillance.

\section{Acknowledgements}

This study was in part supported by JSPS KAKENHI Grant no. 23590480 and MEXT-Supported Program for the Strategic Research Foundation at Private Universities. We thank Mr. Kenta Kobayashi for his technical assistance.

\section{References}

1. Chouaib S, Messai Y, Couve S, Escudier B, Hasmim M and Noman MZ: Hypoxia promotes tumor growth in linking angiogenesis to immune escape. Front Immunol 3: 1-10, 2012.

2. Wilson WR and Hay MP: Targeting hypoxia in cancer therapy. Nat Rev Cancer 11: 393-410, 2011.

3. Höckel M and Vaupel P: Tumor hypoxia: definitions and current clinical, biologic and molecular aspects. J Natl Cancer Inst 93: 266-276, 2001.

4. Melillo G: Inhibiting hypoxia-inducible factor 1 for cancer therapy. Mol Cancer Res 4: 601-605, 2006.

5. Semenza GL: Targeting HIF-1 for cancer therapy. Nat Rev Cancer 3: 721-732, 2003.

6. Semenza GL: Involvement of hypoxia-inducible factor 1 in human cancer. Intern Med 41: 79-83, 2002.

7. Giaccia A, Siim BG and Johnson RS: HIF-1 as a target for drug development. Nat Rev Drug Discov 2: 803-811, 2003.

8. Waldhauer I and Steinle A: NK cells and cancer immunosurveillance. Oncogene 27: 5932-5943, 2008.

9. Nausch $\mathrm{N}$ and Cerwenka A: NKG2D ligands in tumor immunity Oncogene 27: 5944-5958, 2008.

10. Salih HR, Rammensee H-G and Steinle A: Cutting edge: downregulation of MICA on human tumors by proteolytic shedding. J Immunol 169: 4098-4102, 2002.

11. Salih HR, Goehlsdorf D and Steinle A: Release of MICB molecules by tumor cells: mechanism and soluble MICB in sera of cancer patients. Hum Immunol 67: 188-195, 2006.

12. Waldhauer I and Steinle A: Proteolytic release of soluble UL16-binding protein 2 from tumor cells. Cancer Res 66: 2520-2526, 2006.

13. Boutet P, Agüera-González S, Atkinson S, Pennington CJ, Edwards DR, Murphy G, Reyburn HT and Valés-Gómez M: Cutting edge: the metalloproteinase ADAM17/TNF- $\alpha$ converting enzyme regulates proteolytic shedding of the MHC class I-related chain B protein. J Immunol 182: 49-53, 2009.

14. Groh V, Wu J, Yee C and Spies T: Tumour-derived soluble MIC ligands impair expression of NKG2D and T-cell activation. Nature 419: 734-738, 2002.

15. Raffaghello L, Prigione I, Airoldi I, Camoriano M, Levreri I, Gambini C, Pende D, Steinle A, Ferrone S and Pistoia V: Downregulation and/or release of NKG2D ligands as immune evasion strategy of human neuroblastoma. Neoplasia 6: 558-568, 2004.

16. Märten A, von Lilienfeld-Toal M, Büchler MW and Schmidt J: Soluble MIC is elevated in the serum of patients with pancreatic carcinoma diminishing $\gamma \delta \mathrm{T}$ cell cytotoxicity. Int J Cancer 119: 2359-2365, 2006.

17. Lee CT, Mace T and Repasky EA: Hypoxia-driven immunosuppression: a new reason to use thermal therapy in the treatment of cancer? Int J Hyperthermia 26: 232-246, 2010.

18. Siemens DR, Hu N, Sheikhi AK, Chung E, Frederiksen LJ, Pross $\mathrm{H}$ and Graham $\mathrm{CH}$ : Hypoxia increases tumor cell shedding of MHC class I chain-related molecule: role of nitric oxide. Cancer Res 68: 4746-4753, 2008.

19. Barsoum IB, Hamilton TK, Li X, Cotechini T, Miles EA, Siemens DR and Graham CH: Hypoxia induces escape from innate immunity in cancer cells via increased expression of ADAM10: role of nitric oxide. Cancer Res 71: 7433-7441, 2011. 
20. Postovit LM, Adams MA, Lash GE, Heaton JP and Graham CH: Oxygen-mediated regulation of tumor cell invasiveness. Involvement of a nitric oxide signaling pathway. J Biol Chem 277: 35730-35737, 2002.

21. Postovit LM, Sullivan R, Adams MA and Graham CH: Nitric oxide signalling and cellular adaptations to changes in oxygenation. Toxicology 208: 235-248, 2005.

22. Postovit LM, Adams MA, Lash GE, Heaton JP and Graham CH: Nitric oxide-mediated regulation of hypoxia-induced B16F10 melanoma metastasis. Int J Cancer 108: 47-53, 2004.

23. Yoon SY, Lee YJ, Seo JH, Sung HJ, Park KH, Choi IK, Kim SJ, Oh SC, Choi CW, Kim BS, Shin SW, Kim YH and Kim JS: uPAR expression under hypoxic conditions depends on iNOS modulated ERK phosphorylation in the MDA-MB-231 breast carcinoma cell line. Cell Res 16: 75-81, 2006.
24. Quintero M, Brennan PA, Thomas GJ and Moncada S: Nitric oxide is a factor in the stabilization of hypoxia-inducible factor1alpha in cancer: role of free radical formation. Cancer Res 66: 770-774, 2006.

25. Luo L, Lu J, Wei L, Long D, Guo JY, Shan J, Li FS, Lu PY, Li PY and Feng L: The role of HIF-1 in up-regulating MICA expression on human renal proximal tubular epithelial cells during hypoxia/reoxygenation. BMC Cell Biol 11: 1-13, 2010.

26. Wei L, Lu J, Feng L, Long D, Shan J, Li S and Li Y: HIF-1alpha accumulation upregulates MICA and MICB expression on human cardiomyocytes and enhances NK cell cytotoxicity during hypoxia-reoxygenation. Life Sci 87: 111-119, 2010. 